EPJ manuscript No.

(will be inserted by the editor)

\title{
Scaling laws and higher-order effects in Coulomb excitation of neutron halo nuclei
}

\author{
S. Typel ${ }^{1,2}$ and G. Baur ${ }^{3,4}$ \\ 1 Excellence Cluster Universe, Technische Universität München, Boltzmannstraße 2, D-85748 Garching, Germany \\ 2 Gesellschaft für Schwerionenforschung (GSI) mbH, Planckstraße 1, D-64291 Darmstadt, Germany \\ 3 Institut für Kernphysik, Forschungszentrum Jülich, D-52425 Jülich, Germany \\ 4 Jülich Centre for Hadron Physics, Forschungszentrum Jülich, D-52425 Jülich, Germany
}

Received: date / Revised version: date

\begin{abstract}
Essential properties of halo nuclei can be described in terms of a few low-energy constants. For neutron halo nuclei, analytical results can be found for wave functions and electromagnetic transition matrix-elements in simple but well-adapted models. These wave functions can be used to study nuclear reactions; an especially simple and instructive example is Coulomb excitation. A systematic expansion in terms of small parameters can be given. We present scaling laws for excitation amplitudes and cross sections. The results can be used to analyze experiments like ${ }^{11}$ Be Coulomb excitation. They also serve as benchmark tests for more involved reaction theories.
\end{abstract}

PACS. 25.70.De Coulomb excitation - 23.20.Js Multipole matrix elements -27.20.+n $6 \leq A \leq 19$

\section{Introduction}

Exotic nuclei are available as secondary beams at many radioactive beam facilities around the world. These un' stable nuclei are generally weakly bound with few, if any, excited states. A well developed method to study halo nuclei is Coulomb excitation. An instructive example is the excitation of the $1 / 2^{-}$bound state in ${ }^{11} \mathrm{Be}$ from the $1 / 2^{+}$ ground state [1,2,3,4].

Halo nuclei are a low-energy phenomenon and can be described effectively in terms of a few low-energy parameters. The ratios of core size to the sizes of the halo states (to be defined in eq. (11) below) serve as small expansion parameters. Wave functions and matrix elements can be given in terms of these parameters. These wave functions can be used in reaction models and simple and realistic formulae are obtained.

In a recent paper the $B\left(E 1 ; 1 / 2^{+} \rightarrow 1 / 2^{-}\right)$strength for ${ }^{11} \mathrm{Be}$ has been determined from intermediate energy Coulomb excitation measurements [1]. In order to analyse such kind of data in terms of electromagnetic matrix elements it is certainly necessary to use rather sophisticated codes which take higher-order electromagnetic and nuclear effects into account. These codes can be checked and validated by comparing their results to limiting cases where analytical results can be obtained, e.g., pure Coulomb excitation that shows some rather simple features. This can provide a useful guide for more sophisticated approaches. It is the purpose of this paper to provide such analytical

Send offprint requests to: S.Typel results. In the theoretical analysis of [1, quite a complicated XCDCC method was used, which is required for a quantitative description of the experimental data, but it may tend to obscure the understanding of the simple physical mechanism of Coulomb excitation. ${ }^{11} \mathrm{Be}$ is an archetype of a halo nucleus with a ${ }^{10} \mathrm{Be}$ core and a single halo neutron in the $2 s_{1 / 2}$ state. There is a strong $E 1$ transition to the $1 / 2^{-}$bound state, itself a $p$-wave neutron halo state. This dipole transition was previously studied by Coulomb excitation at GANIL, RIKEN, and MSU 2 , 3, 4.

There are two somewhat separated questions: the influence of nuclear excitation in grazing collisions and higherorder electromagnetic effects in distant collisions where nuclear interaction effects can safely be neglected. We deal here with the second question: higher-order electromagnetic excitation. This problem was studied for the ${ }^{11} \mathrm{Be}$ case in the mid-nineties by two groups [5, 6]. In view of recent advances in the description of the electromagnetic properties of halo nuclei, see e.g. [7,8,9,10, it seems appropriate to update this work using the recent analytical results for halo wave functions and present scaling laws for Coulomb excitation of halo nuclei. Effective field theory methods are also applied successfully to halo nuclei, see [11,12].

In section 2 we give the main theoretical formulae for our model of the Coulomb excitation of neutron halo nuclei from $s$ - to $p$-states. In section 3 scaling rules are discussed. Then we give an application to the case of ${ }^{11} \mathrm{Be}$. This can serve as a benchmark for more involved studies like XCDCC or time-dependent approaches [13. Conclu- 
sions are given in section 4. A preliminary account of part of this work was published in the proceedings of the conference 'Nuclear Physics in Astrophysics III' in Dresden, March 2007 [14.

\section{Analytically solvable model for Coulomb excitation of neutron halo nuclei}

A few low-energy parameters are sufficient to characterize halo nuclei. Let us consider the single-particle excitation of a neutron from a ground state $i=0$ to a bound excited state $i=1$ with neutron separation energies $E_{i}>0$. (For ${ }^{11} \mathrm{Be}$ we have $E_{0}=504 \mathrm{keV}$ and $E_{1}=184 \mathrm{keV}$.) The size of the single-particle wave functions is determined by the bound-state constants $q_{i}=\sqrt{2 \mu E_{i}} / \hbar$ with the reduced mass $\mu=m_{n} m_{c} /\left(m_{n}+m_{c}\right)$ of the nucleon+core system. With the radius $R$ of the core beyond which the nuclear interaction is assumed to vanish, it is possible to introduce dimensionless parameters

$$
\gamma_{i}=q_{i} R
$$

that are a measure for the ratio of the core size to the size of the neutron wave functions. These parameters are small for halo nuclei, see, e.g., 7,8, and can be used as convenient expansion parameters. In this spirit, we use this single-particle model for the $j_{i}^{\pi}=1 / 2^{+}$and $j_{f}^{\pi}=$ $1 / 2^{-}$states in order to evaluate the matrix element for the $E 1$ electromagnetic excitation. It is dominated by the exterior contributions.

\subsection{Halo wave functions and matrix elements}

The single-particle wave functions are given by

$$
\Phi_{i}(\boldsymbol{r})=\frac{f_{i}(r)}{r} \mathcal{Y}_{j_{i} m_{i}}^{l_{i} s}(\hat{r})
$$

for the ground state $(i=0)$ and the excited state $(i=$ $1)$. The angular dependence is described by the spinor spherical harmonics $\mathcal{Y}_{j m}^{l s}(s=1 / 2)$. We use the results and notation of $[8]$ assuming zero spin of the core. The radial wave functions of the two states are given in the exterior region $(r>R)$ by

$$
f_{0}(r)=C_{0} q_{0} r h_{0}^{(1)}\left(i q_{0} r\right)=-C_{0} \exp \left(-q_{0} r\right)
$$

and

$$
\begin{aligned}
f_{1}(r) & =C_{1} i q_{1} r h_{1}^{(1)}\left(i q_{1} r\right) \\
& =-C_{1} \exp \left(-q_{1} r\right)\left(1+\frac{1}{q_{1} r}\right)
\end{aligned}
$$

with Hankel functions $h_{l}^{(1)}$ of imaginary argument. Both states are halo states and the normalization constants are given in the halo limit by $C_{0}=\sqrt{2 q_{0}}$ and $C_{1}=\sqrt{2 q_{1}^{2} R / 3}$, respectively $[8]$.
The parameters $q_{0}$ and $q_{1}$ of the shallow bound states in a halo nucleus are closely related to the scattering length $a_{l}$ and the effective range parameter $r_{l}$ for partial waves $l \geq 0$ in the effective range expansion $k^{2 l+1} \cot \left(\delta_{l}\right)=$ $-1 / a_{l}+r_{l} k^{2} / 2+\ldots$. The S matrix $S_{l}=\left[\cot \left(\delta_{l}\right)+i\right] /\left[\cot \left(\delta_{l}\right)-\right.$ $i]$ has a pole at $k=i k_{B}$, i.e. $\cot \left[\delta_{l}\left(k_{B}\right)\right]=i$ for a bound state with $k_{B}=q_{i}$. This gives the desired relation

$$
(-1)^{l+1} q_{l}^{2 l+1}=-\frac{1}{a_{l}}-\frac{1}{2} r_{l} q_{l}^{2}+\ldots .
$$

Now there is a difference between $l=0$ and $l>0$. For $l=0$ and small $q_{0}$ we have in lowest order the relation $q_{0}=1 / a_{0}$ and the effective-range term is a small correction. For $l>0$ the left hand side of (5) is smaller than the first two terms individually on the right hand side and we have an enhanced value (as compared to "dimensional considerations") of the scattering length that is given in lowest order by $a_{l}=-2 /\left(r_{l} q_{l}^{2}\right)$ ("fine tuned"). In [7] the scattering length in the $l=1, j=1 / 2$ channel of ${ }^{11} \mathrm{Be}$ was determined to be $457(67,-66) \mathrm{fm}^{3}$. With $q_{1}=0.0895 \mathrm{fm}^{-1}$ in this channel we find an effective-range parameter $r_{1}=-0.547 \mathrm{fm}^{-1}$ of "natural order" $R^{-1}$.

With the radial wave functions (3) and (4) we calculate the $B(E 1)$ value for the $1 / 2^{+} \rightarrow 1 / 2^{-}$-transition as well as the higher-order effects in electromagnetic excitation. We propose this to be a model study and leave the spectroscopic factors equal to one. (They could be adjusted, which would result in a quasi-realistic description of the ${ }^{11} \mathrm{Be}$ system for our purpose.) The $B(E 1)$ value is given by

$$
B(E 1)=\frac{1}{4 \pi}\left(Z_{\mathrm{eff}}^{(1)} e\right)^{2}\left|R_{01}^{(1)}\right|^{2},
$$

where $Z_{\text {eff }}^{(1)}=Z_{c} m_{n} /\left(m_{n}+m_{c}\right)$ is the dipole effective charge number and the radial dipole integral is given by

$$
\begin{aligned}
R_{01}^{(1)} & =\int_{0}^{\infty} d r f_{1}^{*}(r) r f_{0}(r) \\
& =2 \sqrt{\frac{\gamma_{0}}{3}} \frac{\left(\gamma_{0}+2 \gamma_{1}\right)}{\left(\gamma_{0}+\gamma_{1}\right)^{2}} R .
\end{aligned}
$$

In the present paper we are only interested in the halo limit, i.e., we keep only the lowest-order term in the expansion in $\gamma_{0}$ and $\gamma_{1}$. In this approximation we can use the exterior radial wave functions, eqs. (3) and (4) in the integral eq. (77) down to $r=0$. The correction terms from using the correct interior wave functions are of higher order in the expansion parameters $\gamma_{0}$ and $\gamma_{1}$. For $R \rightarrow 0$ the radial dipole integral goes to zero because the normalization of the $p$-wave function tends to zero in this limit. Thus $R$ must be kept finite; we choose $R=2.78 \mathrm{fm}$ as a realistic value for ${ }^{11} \mathrm{Be}[7$. This value determines the asymptotic normalization of the $p$-wave bound state. We find $B(E 1)=0.193 \mathrm{e}^{2} \mathrm{fm}^{2}$, to be compared to the value of $B(E 1)=0.105(12) \mathrm{e}^{2} \mathrm{fm}^{2}$ obtained from an analysis of the GANIL data, see [1. This value is consistent with other Coulomb dissociation experiments at RIKEN and MSU and the value obtained by the Doppler shift attenuation method [15]. 


\subsection{Coulomb excitation of neutron halo nuclei and scaling laws}

We treat electromagnetic excitation in the semiclassical approximation. For high beam energies the classical trajectory can be taken as a straight line with impact parameter $b$. In the sudden approximation one can take higherorder effects into account in a convenient way. Like the related Glauber approximation it is applicable for high beam energies and low excitation energies. This is reasonably well fulfilled for ${ }^{11} \mathrm{Be}$, even at GANIL energies. In [5] higher-order effects in the electromagnetic excitation of ${ }^{11}$ Be to the $1 / 2^{-}$bound state were studied using the sudden approximation. In [6] a continuum discretized coupled channels approach was adopted. In [16] an analytically solvable model for higher-order effects in the electromagnetic dissociation of neutron halo nuclei was presented. In that work, there was only the transition from an $s$-wave bound state to the continuum. Now we consider the case where there is, in addition, a $p$-wave bound state, as it is the case in ${ }^{11} \mathrm{Be}$. In the sudden approximation the dipole excitation amplitude from the ground state $i$ to the final state $f$ is given by

$$
a_{\text {sudden }}=\left\langle f\left|\exp \left(-i \boldsymbol{q}_{\text {Coul }} \cdot \boldsymbol{r}\right)\right| i\right\rangle
$$

where the transfered momentum is

$$
\hbar \boldsymbol{q}_{\mathrm{Coul}}=\frac{2 Z Z_{\mathrm{eff}}^{(1)} e^{2}}{v b} \boldsymbol{e}_{z} .
$$

The impact parameter $b$ is chosen to be in the $z$-direction; this is convenient for the following calculation using polar coordinates. The target charge number is denoted by $Z$ and $v$ is the beam velocity. The dipole approximation is quite well fulfilled, since the dipole effective charge $Z_{\text {eff }}^{(1)}$ is much larger than the corresponding quadrupole charge. The neutron and core masses are denoted by $m_{n}$ and $m_{c}$ respectively, the charge of the core is given by $Z_{c}$. The sudden approximation is applicable for $\xi \equiv \omega b / v \ll 1$, where $\hbar \omega=E_{0}-E_{1}$ is the excitation energy. Even for the comparatively low GANIL energies of about $40 \mathrm{MeV} /$ nucleon this is reasonably well fulfilled. The most important intermediate states are expected to be in the low-energy continuum, where the dipole strength has a peak, at around $1 \mathrm{MeV}$ excitation energy. Thus the adiabaticity condition $\xi \ll 1$ will also apply to the important intermediate states. We note that for the virtual excitation of the high-lying giant dipole state the opposite limit $\xi \gg 1$ is realized, this would lead to a real polarization potential, see e.g. [17]. It influences the classical trajectory of the projectile and it is not important in our context. It is also neglected in our model space. The sudden approximation has the advantage that intermediate states are treated by closure, thus one only needs a model for the initial and final states, and not for all the intermediate states. In lowest order in $q_{\text {Coul }}$ the first-order dipole approximation is obtained corresponding to a single-photon exchange between target and projectile. It is shown in [5] that third-order $E 1$ excitation is more important than second-order E1-E2 excitation. The role of higher multipole excitations in the electromagnetic excitation of one-neutron halo nuclei is also studied in 18 .

In the appendix we show that

$$
a_{\text {sudden }}=(-1)^{j_{i}+m_{i}} \frac{C_{0} C_{1}}{2 i}\left[\frac{1}{q_{\text {Coul }}} I_{1}(z)+\frac{1}{q_{1}} I_{2}(z)\right]
$$

where

$$
I_{1}(z)=2\left[1-\frac{\arctan (z)}{z}\right]
$$

and

$$
I_{2}(z)=\left(1+\frac{1}{z^{2}}\right) \arctan (z)-\frac{1}{z}
$$

depending on the dimensionless parameter

$$
z=\frac{q_{\mathrm{Coul}}}{q_{0}+q_{1}}=\frac{q_{\mathrm{Coul}} R}{\gamma_{0}+\gamma_{1}} .
$$

This quantity is proportional to the usual strength parameter

$$
\chi^{(1)}=\frac{Z e\langle f|| \mathcal{M}(E 1) \| i\rangle}{\hbar v b}
$$

that characterizes the importance of higher-order effects in Coulomb excitation processes. We note that this parameter becomes large in the halo limit $(\gamma \rightarrow 0)$. In contrast to $\chi^{(1)}$, the adiabaticity parameter $\xi=\hbar \omega /(v b)$ with the excitation energy $\hbar \omega=\hbar^{2}\left(q_{0}^{2}-q_{1}^{2}\right) /(2 \mu)$ becomes small in the halo limit and the application of the sudden approximation (8) is very well justified.

We can expand the excitation amplitude (10) in powers of $z$ with the help of the power series of the arctan function. This power series expansion converges for $|z| \leq 1$ and one obtains

$$
a_{\text {sudden }}=a_{1}+a_{3}+\ldots
$$

with the lowest-order term

$$
a_{1}=(-1)^{j_{i}+m_{i}} \frac{2}{3 i} \sqrt{\frac{\gamma_{0}}{3}} \frac{\gamma_{0}+2 \gamma_{1}}{\gamma_{0}+\gamma_{1}} z
$$

and the next-to-leading order term

$$
a_{3}=(-1)^{j_{i}+m_{i}} \frac{2 i}{15} \sqrt{\frac{\gamma_{0}}{3}} \frac{\gamma_{0}+4 \gamma_{1}}{\gamma_{0}+\gamma_{1}} z^{3} .
$$

The even terms vanish due to the parity selection rule. Note that in the sudden approximation (8) there is no change in the magnetic quantum number, since the vector $\boldsymbol{q}_{\text {Coul }}$ is in the $z$-direction.

Following Ref. [5] we can introduce a reduction factor

$$
r(z)=\frac{\left|a_{\text {sudden }}\right|^{2}}{\left|a_{1}\right|^{2}} \approx 1-\frac{2}{5} \frac{\gamma_{0}+4 \gamma_{1}}{\gamma_{0}+2 \gamma_{1}} z^{2}+\ldots
$$

In figure 1 the dependence of the reduction factor $r$ on $z$ is shown for the present case with $\gamma_{1} / \gamma_{0}=\sqrt{E_{1} / E_{0}} \approx 0.604$ by the solid line. We find $r(z) \approx 1-0.619 z^{2}$ in lowest order in $z$. It seems of interest to compare the results of [5] with the present one. In [5] two different models (denoted by $I$ and $I I$ ) were used. Expanding in the 


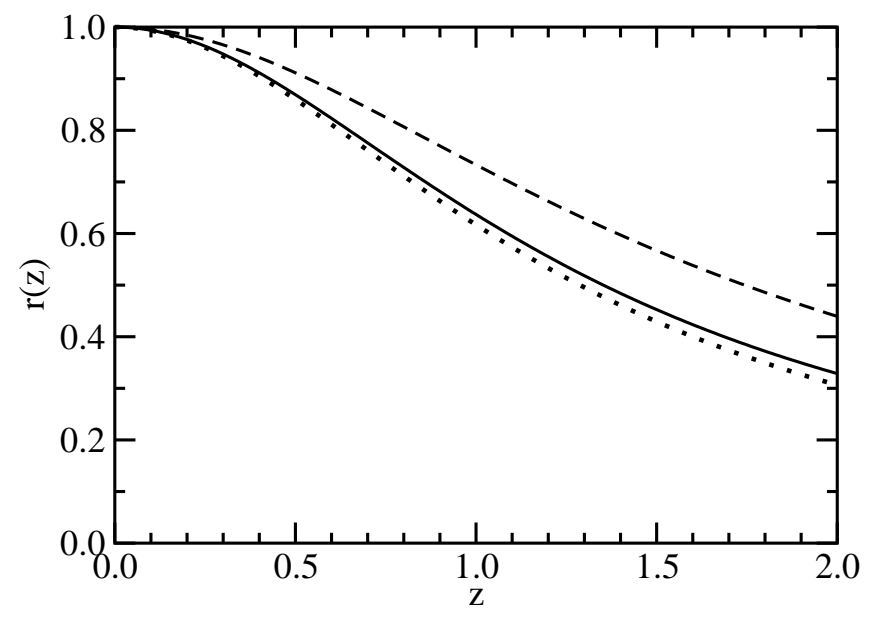

Fig. 1. Reduction factor $r(z)=\left|a_{\text {sudden }}\right|^{2} /\left|a_{1}\right|^{2}$ as a function of the scaling parameter $z$ as defined in eq. (13) for values of 0 (dashed line), 0.604 (solid line) and 1 (dotted line) of the ratio $\gamma_{1} / \gamma_{0}=\sqrt{E_{1} / E_{0}}$

strength parameter $z$ we get $r^{I}(z)=1-6 z^{2} / 5+\ldots$, and $r^{I I}(z)=1-12 z^{2} / 5+\ldots$ The present higher-order corrections are smaller than the one found in [5]. In that reference rather crude models were used for the first excited $1 / 2^{-}$-state; those wave functions extended much further out than the present wave function (4), which takes the angular momentum barrier into account properly. In the higher-order terms the importance of the outer region is enhanced due to the weighting with a higher power of $\mathrm{r}$. It should be recalled that the main aim of [5] was to provide an upper limit for higher-order effects in order to understand the results of [2]. In the meantime, other Coulomb excitation experiments were performed [1,3,4 that clarified the situation.

In the general case of a Coulomb excitation from a bound $s$-wave to a bound $p$-wave neutron halo state, the energy $E_{1}$ of the excited state is limited from below by zero (state at the breakup threshold) and from above by $E_{0}$ (zero-energy excitation). This corresponds to the intervall $[0,1]$ for the ratio $\gamma_{1} / \gamma_{0}$. The two limiting cases are depicted in figure 1 by dashed and dotted lines.

The excitation probability is given by

$$
\begin{aligned}
P(b) & =\frac{1}{2 j_{i}+1} \sum_{m_{i} m_{f}} \delta_{m_{i} m_{f}}\left|a_{\text {sudden }}\right|^{2} \\
& =P_{\mathrm{LO}}(b)+P_{\mathrm{NLO}}(b)+\ldots
\end{aligned}
$$

The lowest-order term

$$
P_{\mathrm{LO}}(b)=\frac{4 \gamma_{0}\left(\gamma_{0}+2 \gamma_{1}\right)^{2}}{27\left(\gamma_{0}+\gamma_{1}\right)^{2}} z^{2} \equiv \frac{A_{2}}{b^{2}}
$$

in the excitation probability is proportional to $z^{2}$. The most important higher-order contribution comes from $a_{3}$. Its interference with the lowest-order term $a_{1}$ leads to the next term

$$
P_{\mathrm{NLO}}(b)=-\frac{8 \gamma_{0}\left(\gamma_{0}+2 \gamma_{1}\right)\left(\gamma_{0}+4 \gamma_{1}\right)}{135\left(\gamma_{0}+\gamma_{1}\right)^{2}} z^{4} \equiv-\frac{A_{4}}{b^{4}}
$$

in the expansion of $P(b)$ in $z$, of the order of $z^{4}$. The constants $A_{2}$ and $A_{4}$ can be written as

$$
A_{2}=\frac{4 \gamma_{0}\left(\gamma_{0}+2 \gamma_{1}\right)^{2}}{27\left(\gamma_{0}+\gamma_{1}\right)^{4}}\left(\frac{2 \eta m_{n}}{m_{c}+m_{n}}\right)^{2} R^{2}
$$

and

$$
A_{4}=\frac{8 \gamma_{0}\left(\gamma_{0}+2 \gamma_{1}\right)\left(\gamma_{0}+4 \gamma_{1}\right)}{135\left(\gamma_{0}+\gamma_{1}\right)^{6}}\left(\frac{2 \eta m_{n}}{m_{c}+m_{n}}\right)^{4} R^{4}
$$

where the Coulomb parameter is given by $\eta=Z Z_{c} e^{2} /(\hbar v)$. Total cross sections are obtained by integration over the impact parameter, starting from a minimum impact parameter $b_{\min }$. The sudden approximation fails for large impact parameters, and an adiabatic cut-off $b_{\max }=\gamma_{\text {beam }} v / \omega$ with the Lorentz-factor by $\gamma_{\text {beam }}=1 / \sqrt{1-(v / c)^{2}}$ has to be introduced for the lowest-order result. For the higherorder terms this is not necessary, the convergence in $b$ is fast enough. We get

$$
\sigma_{\mathrm{LO}}=2 \pi \int_{b_{\min }}^{b_{\max }} P_{L O}(b) b d b=2 \pi A_{2} \ln \frac{b_{\max }}{b_{\min }}
$$

and

$$
\sigma_{\mathrm{NLO}}=2 \pi \int_{b_{\min }}^{\infty} P_{N L O}(b) b d b=-\frac{\pi A_{4}}{b_{\min }^{2}} .
$$

(A somewhat different method was used in eqs. (16) and (17) of [5], where the $\xi$-dependence of the first-order amplitude $a_{1}$ is taken into account. Then there is no need for the adiabatic cutoff $b_{\max }$.)

We note that the strength parameter $z$ is proportional to $1 / v$, i.e. the leading-order term decreases like $1 / E$, the next-to-leading-order term like $1 / E^{2}$, where $E$ is the beam energy. In the halo limit $\left(\gamma \equiv \gamma_{0}, \gamma_{1} \rightarrow 0\right)$ the probabilities scale as $P_{\mathrm{LO}} \propto 1 / \gamma$ and $P_{\mathrm{NLO}} \propto 1 / \gamma^{3}(z$ scales as $1 / \gamma)$ and the excitation probability tends to infinity. However, the NLO contribution tends to infinity even faster and higherorder corrections become more important. Still, for realistic values of $\gamma$ the higher order effects are quite small (see below). It seems intuitively understandable that higherorder effects tend to increase with decreasing $\gamma$. In fact, this increase with decreasing $\gamma$ is faster than the increase in lowest order, as our analysis shows.

For the total cross section there is the additional well known enhancement $\ln \gamma$ in LO, which is absent in NLO, due to the fast convergence in $b$.

In [16] and [19] the scaling properties of the Coulomb dissociation of halo nuclei were investigated in a related approach. The strength parameter defined there corresponds to the one defined now. Using the present notation we found in that case that $P_{\mathrm{LO}} \propto 1 / \gamma^{3}$ and $P_{\mathrm{NLO}} \propto$ $1 / \gamma^{5}$. The difference to the present case arises because the bound-continuum and bound-bound state dipole matrix elements show different scaling properties with $\gamma_{i}$.

\section{Application to the ${ }^{11} \mathrm{Be}$ Coulomb excitation experiments}

In the case of ${ }^{11} \mathrm{Be}$ we have neutron separation energies of $E_{0}=504 \mathrm{keV}$ and $E_{1}=184 \mathrm{keV}$ for the ground 
Table 1. Projectile energy per nucleon $E / A$, velocity $v$, maximum impact parameter $b_{\max }$, maximum parameter $z_{\max }$ and cross section reduction factor $R(v)$ for the ${ }^{11}$ Be experiments.

\begin{tabular}{lccccc}
\hline & $\begin{array}{c}E / A \\
{[\mathrm{MeV}]}\end{array}$ & $\begin{array}{c}v \\
{[c]}\end{array}$ & $\begin{array}{c}b_{\max } \\
{[\mathrm{fm}]}\end{array}$ & $z_{\max }$ & $R(v)$ \\
\hline GANIL [1] & 38.6 & 0.2861 & 184.1 & 0.6549 & 0.8902 \\
GANIL [2] & 43.0 & 0.3009 & 194.6 & 0.6227 & 0.9025 \\
MSU [4] & 60.0 & 0.3507 & 230.9 & 0.5343 & 0.9321 \\
RIKEN [3] & 64.0 & 0.3611 & 238.8 & 0.5189 & 0.9366 \\
\hline
\end{tabular}

and excited bound state. This leads to an excitation energy of $\hbar \omega=320 \mathrm{keV}$ for the $1 / 2^{-}$state. With a radius $R=2.78$ fm one obtains the numerical values $\gamma_{0}=0.4116$ and $\gamma_{1}=0.2487$, respectively, for the dimensional scaling parameters that appear in the excitation amplitude. Depending on the projectile energy, the projectile velocities $v$ and the maximum impact parameters $b_{\max }=\gamma_{\text {beam }} v / \omega$ are found for the conditions of the experiments at GANIL [1, 2, RIKEN 3] and MSU [4. They are given in table 1. These adiabatic cutoff radii are impressively large. It means that Coulomb excitation extends really far out and there is an amply large region where the interaction is purely electromagnetic, and our approach works very transparently. Of course, there is a Coulomb and nuclear interference zone, quite moderate in extension, close to the minimum impact parameter $b_{\min }=1.2\left(11^{1 / 3}+\right.$ $\left.208^{1 / 3}\right) \mathrm{fm}=9.78 \mathrm{fm}$ which is not considered in our approach.

The maximum value of the dimensionless strength parameter (13) is given by $z_{\max }=2 Z Z_{\mathrm{eff}}^{(1)} e^{2} R /\left[\hbar v b_{\min }\left(\gamma_{0}+\right.\right.$ $\left.\gamma_{1}\right)$ ] with the target charge number $Z=82$ and the effective charge number $Z_{\text {eff }}^{(1)}=4 / 11$. The numerical values for $z_{\text {max }}$ are given again in table 1. They are smaller than one and decrease with the projectile energy. The range where the reduction factor is actually needed is well approximated by the NLO correction, which corresponds to the approximation of $r(z)$ in figure 1 by an inverted parabola. In the present approach we are more certain about the nuclear structure input that determines the opening parameter of this parabola.

Similar as in [5] we define a reduction factor $R(v)=$ $\sigma^{(\infty)} / \sigma_{L O}$ of the total excitation cross section $\sigma^{(\infty)}$ caused by higher-order effects. This factor $R(v)$ can be used to take higher order effects into account in an analysis of the experimental data.

Since the parameter $z$ is always smaller than one in the experiments, the full cross section in sudden approximation $\sigma^{(\infty)}=\sigma_{L O}+\sigma_{N L O}+\ldots$ is well approximated by the NLO correction and the reduction factor becomes

$$
R(v) \approx 1+\frac{\sigma_{N L O}}{\sigma_{L O}}=1-\frac{A_{4}}{2 A_{2} b_{\min }^{2} \ln \frac{b_{\max }}{b_{\min }}}
$$

The numerical values as given in table 1 show that the reduction of the first-order cross section is in the order of 6 to $11 \%$ for the various experiments. In figure 2 the dependence of the reduction on the projectile velocity $v$

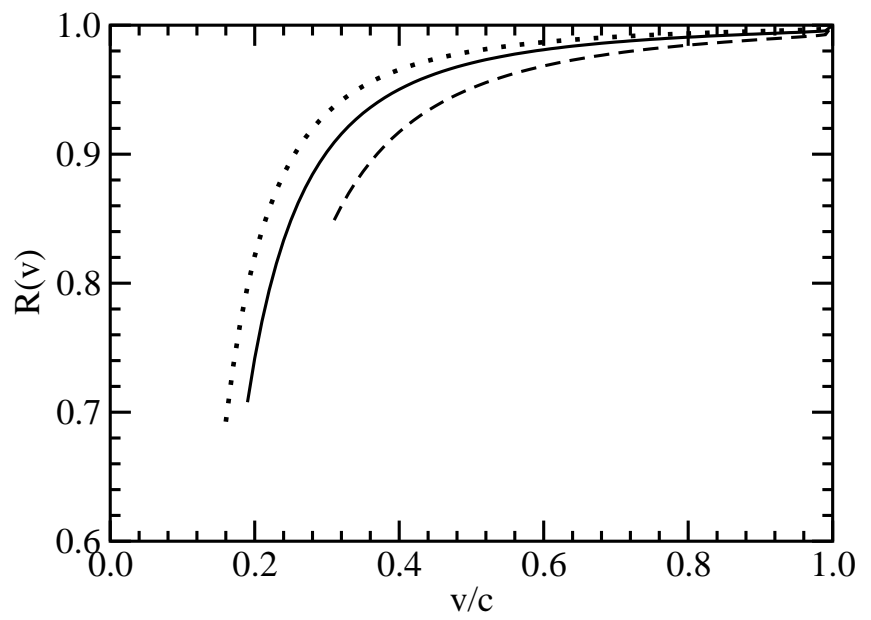

Fig. 2. Reduction factor $R(v)=1+\sigma_{N L O} / \sigma_{L O}$ as a function of the velocity $v$ for values of 0 (dashed line), 0.604 (solid line) and 1 (dotted line) of the ratio $\gamma_{1} / \gamma_{0}=\sqrt{E_{1} / E_{0}}$.

is depicted for the actual value of the ratio $\gamma_{1} / \gamma_{0}$ and the two limiting cases as in figure 11. The curves are shown only for velocities where the parameter $z$ does not exceed the value one, the radius of convergence for the expansion of the amplitude (15). Note that an increase of $\gamma_{1}$ from zero (dashed line) to one (dotted line) leads to a reduction of the parameter $z$ and correspondingly to a reduction of the higher-order corrections in the cross section for the same velocity $v$.

\section{Conclusion}

We have presented a realistic model for higher-order effects in the Coulomb excitation of neutron halo nuclei. With quite simple methods a reliable value for the reduction of the cross section could be given. In addition, this should be very useful as a benchmark, for valuable and necessary tests of more sophisticated approaches like CDCC or time-dependent calculations. These kinds of calculations require a lot of computation, yet they should reproduce the present results in certain well defined limits. Using an effective-range point of view we updated our previous work 5. Other angular momentum combinations can be treated in a similar way. However, for higher angular momenta the halo nature is less pronounced and the importance of the core size parameter $\mathrm{R}$ increases. Higher-order terms in $\gamma$ will become more important and the method less useful. It is quite remarkable that our results for ${ }^{11} \mathrm{Be}$ Coulomb excitation depend only on the binding energies (they determine the parameters $q_{0}$ and $q_{1}$ ) and a parameter $R$ which characterizes the core size. This stresses the fact that halo nuclei depend only on a few low-energy constants, and not on details of the shape of the potential. This is in accord with low-energy scattering, which is also characterized by a few low-energy parameters in the effective-range expansion.

In view of the discovery of the neutron-rich isotopes ${ }^{40} \mathrm{Mg}$ and ${ }^{42} \mathrm{Al}$ [20,21] one may expect that more neutron- 
rich nuclei with low-lying single particle states exist to which the present approach can be applied. It is very likely that the new radioactive beam facilities around the world like FAIR, SPIRAL2 and RIBF will provide interesting new examples in the higher mass region.

\section{Acknowledgment}

This research was supported by the DFG cluster of excellence "Origin and Structure of the Universe".

\section{Appendix}

For the analytical calculation of the excitation amplitude in sudden approximation the argument of the exponential function in (8) can be written as $\boldsymbol{q}_{\text {Coul }} \cdot \boldsymbol{r}=q_{\mathrm{Coul}} r \cos (\theta)$ in spherical coordinates because the vector $\boldsymbol{q}_{\text {Coul }}$ points in $z$-direction. Using

$$
\left[\mathcal{Y}_{j_{f} m_{f}}^{l_{f} s}(\hat{r})\right]^{\dagger} \mathcal{Y}_{j_{i} m_{i}}^{l_{i} s}(\hat{r})=(-1)^{j_{i}+m_{i}} \delta_{m_{i} m_{f}} \frac{\cos (\theta)}{4 \pi}
$$

for $j_{i}=j_{f}=\left|m_{i}\right|=\left|m_{f}\right|=\frac{1}{2}, l_{i}=0, l_{f}=1$ and $s=1 / 2$, one finds

$$
\begin{aligned}
& a_{f i} \\
& =(-1)^{j_{i}+m_{i}} \frac{C_{0} C_{1}}{4 \pi} \int_{0}^{\infty} d r \int_{0}^{2 \pi} d \phi \int_{-1}^{1} d \cos (\theta) \cos (\theta) \\
& \quad \times \exp \left[-\left(q_{1}+q_{0}\right) r\right]\left(1+\frac{1}{q_{1} r}\right) \exp \left[-i q_{\text {Coul }} r \cos (\theta)\right] \\
& =(-1)^{j_{i}+m_{i}} \frac{C_{0} C_{1}}{2 i}\left(\frac{1}{q_{\text {Coul }}} I_{1}+\frac{1}{q_{1}} I_{2}\right)
\end{aligned}
$$

with two integrals that can be calculated separately. The first integral is given by

$$
\begin{aligned}
I_{1}(p, q) & =i q \int_{0}^{\infty} d r \int_{-1}^{1} d z z \exp [-(p+i q z) r] \\
& =i q \int_{-1}^{1} d z \frac{z}{p+i q z} \\
& =2-\frac{p}{i q} \ln \frac{p+i q}{p-i q}
\end{aligned}
$$

with $p=q_{0}+q_{1}$ and $q=q_{\text {Coul }}$. With the relation

$$
\ln \frac{1+i z}{1-i z}=2 i \arctan (z)
$$

one obtains

$$
I_{1}(p, q)=2\left[1-\frac{p}{q} \arctan \left(\frac{q}{p}\right)\right] .
$$

The second integral is given by

$$
\begin{aligned}
I_{2}(p, q) & =i \int_{0}^{\infty} d r \int_{-1}^{1} d z z \frac{\exp [-(p+i q z) r]}{r} \\
& =-\frac{d}{d q} \int_{0}^{\infty} d r \int_{-1}^{1} d z \frac{\exp [-(p+i q z) r]}{r^{2}} \\
& =2 \int_{0}^{\infty} d r \frac{\exp (-p r)}{r} j_{1}(q r)
\end{aligned}
$$

with a spherical Bessel function $j_{1}(x)=-\frac{d}{d x} j_{0}(x)$ so that

$$
\lim _{q \rightarrow 0} I_{2}(p, q)=0 \text {. }
$$

We notice

$$
\frac{d}{d p} I_{2}(p, q)=-\frac{1}{q} I_{1}(p, q)
$$

and find with the help of a partial integration

$$
\begin{aligned}
I_{2}(p, q)= & C-\frac{1}{q} \int_{0}^{p} d p^{\prime} I_{1}\left(p^{\prime}, q\right) \\
= & C-2 \frac{p}{q}+\frac{p^{2}}{q^{2}} \arctan \left(\frac{q}{p}\right) \\
& +\frac{p}{q}-\arctan \left(\frac{p}{q}\right)
\end{aligned}
$$

where a constant $C$ appears. Since

$$
\arctan (x)+\arctan \left(\frac{1}{x}\right)=\frac{\pi}{2}
$$

for $x \geq 0$ we can write

$$
I_{2}(p, q)=C-\frac{p}{q}+\left(1+\frac{p^{2}}{q^{2}}\right) \arctan \left(\frac{q}{p}\right)-\frac{\pi}{2} .
$$

With

$$
\lim _{q \rightarrow 0} I_{2}(p, q)=C-\frac{\pi}{2}=0
$$

the constant can be determined to be $C=\pi / 2$ and the final result is

$$
I_{2}(p, q)=\left(1+\frac{p^{2}}{q^{2}}\right) \arctan \left(\frac{q}{p}\right)-\frac{p}{q} .
$$

\section{References}

1. N.C. Summers et al., Phys. Lett. B 650, 124 (2007).

2. R. Anne et al., Z. Phys. A 352, 397 (1995).

3. T. Nakamura et al., Phys. Lett. B 394, 11 (1997).

4. M. Fauerbach et al., Phys. Rev. C 56, R1 (1997).

5. S. Typel and G. Baur, Phys. Lett. B 356, 186 (1995).

6. C.A. Bertulani, L.F. Canto and M.S. Hussein, Phys. Lett. B 353, 413 (1995).

7. S. Typel and G. Baur, Phys. Rev. Lett. 93142502 (2004).

8. S. Typel and G. Baur, Nucl. Phys. A 759, 247 (2005).

9. M.A. Nagarajan, S.M. Lenzi and A. Vitturi, Eur. Phys. J. A 24, 63 (2005).

10. Z. H. Liu, X. Z. Zhang and H. Q. Zhang, Phys. Rev. C 68, 024305 (2003).

11. P. F. Bedaque, H.-W. Hammer and U. van Kolck, Phys. Lett. B 569, 159 (2003).

12. C. A. Bertulani, H.-W. Hammer and U. van Kolck, Nucl. Phys. A 712, 37 (2002).

13. G. Goldstein, P. Capel and D. Baye, Phys. Rev. C 76, 024608 (2007); G. Goldstein, P. Capel and D. Baye, Phys. Rev. C 73, 024602 (2006); P. Capel, D. Baye and V. S. Melezhik, Phys. Rev. C 68, 014612 (2003) and further references given there. 
14. G. Baur and S. Typel, J. Phys. G: Nucl. Part. Phys. 35, 0140218 (2008).

15. D. J. Millener et al., Phys. Rev. C 28, 497 (1983).

16. S. Typel and G.Baur, Phys. Rev. C 64, 0246101 (2001).

17. A. Winther and K. Alder, Electromagnetic Excitation (North-Holland, Amsterdam 1975).

18. R. Chatterjee, L. Fortunato and A. Vitturi, Eur. Phys. J. A 35, 213 (2008).

19. S. Typel and G.Baur, Nucl. Phys. A 573, 486 (1994).

20. T. Baumann et al., Nature 449, 1022 (2007).

21. P.-H. Heenen, Nature 449, 992 (2007). 